\title{
Portage nasal de Staphylococcus aureus résistant à la méticilline chez des volontaires sains et des malades hospitalisés à Ouagadougou, Burkina Faso
}

\section{Nasal carriage of methicillin-resistant Staphylococcus aureus in healthy volunteers and hospitalized patients in Ouagadougou, Burkina Faso}

\author{
Soré $\mathrm{S}^{1,2}$, Sawadogo $\mathrm{Y}^{3}$., Sanou $\mathrm{S}^{3}$, Béogo $\mathrm{S}^{1}$, Dakouo $\mathrm{SNP}^{3}$, Djamalladine MD ${ }^{4}$, Ilboudo-Koui.S ${ }^{1}$, Zoungrana \\ $\mathrm{J}^{3}$, Poda $\mathrm{A}^{3}$, Ouédraogo $\mathrm{AS}^{3}$, Sanou $\mathrm{I}^{1,2}$. \\ ${ }^{1}$ Centre hospitalier universitaire de Tengandogo, Ouagadougou, Burkina Faso \\ ${ }^{2}$ Université Saint Thomas d'Aquin, Ecole doctorale des sciences, santé et Technologies \\ ${ }^{3}$ Centre hospitalier universitaire Souro Sanou, Bobo-Dioulasso, Burkina Faso \\ ${ }^{4}$ Institut national supérieur des sciences et technique d'Abéché, Abéché, Tchad
}

Auteur correspondant : Soré Souleymane; Direction des laboratoires de biologie médicale, Ministère de la santé, 03 BP 7022 Ouagadougou 03, Burkina Faso. email: soulsore11@yahoo.fr

\begin{abstract}
RESUMÉ
Objectifs: Le but de cette étude était de déterminer la prévalence du portage de Staphylococcus aureus résistant à la méticilline (SARM), d'identifier les types de résistances et d'en analyser les facteurs. Matériel et méthodes: Une étude transversale a été menée auprès des patients et des volontaires. Après leur consentement éclairé, des écouvillonnages nasaux réalisés chez des patients ont permis d'isoler et d'identifier les souches de Staphylococcus aureus par la méthode biochimique. La résistance à la méticilline et les résistances associées ont été déterminés par la méthode de diffusion des disques selon la norme du comité de l'antibiogramme de la société française de microbiologie. Résultats: Sur un total de 189 échantillons analysés ont permis d'isoler 10 SARM soit une prévalence globale de 5,29\% (7,95\% chez les patients et 2,97\% parmi les volontaires) $(p=0,24)$. La fréquence de SARM parmi les $S$. aureus a été de $27,77 \%$ dont $31,81 \%$ chez les patients et $21,42 \%$ chez les volontaires. Les SARM ont été résistants à $100 \%$ et $66,67 \%$ à la norfloxacine et à $57,14 \%$ et $33,33 \%$ à l'érythromycine respectivement chez les patients et chez les volontaires. Cependant la gentamicine a enregistré une sensibilité de $100 \%$. Le sexe, l'âge, la prise antérieure d'antibiotiques n'étaient pas associés au portage de SARM. Conclusion : Cette étude sur la prévalence de Staphylococcus aureus résistant à la méticilline rapporte des résultats inquiétants. Il y a une urgence à développer les mesures de surveillance de la diffusion de ces souches multirésistantes. Mots clé : Ouagadougou, patients hospitalisés, portage, SARM, volontaires sains.
\end{abstract}

\begin{abstract}
Objectives: The purpose of this study was to determine the prevalence of carrying methicillin-resistant Staphylococcus aureus (MRSA), to identify the associated resistances and to analyze the factors. Materials and methods: A cross-sectional study was conducted with patients and volunteers. After their informed consent, nasal swabs performed in patients which is use to isolate and identify Staphylococcus aureus by cultural and biochemical characteristics. The resistance to methicillin and the associated resistances were determined by testing the susceptibility of the strains by the disc diffusion method according to the recommendations of committee of the French Society of Microbiology. Results: From 189 samples we isolated 10 MRSA, an overall prevalence of $5.29 \%$ (7.95\% in hospitalized patients and $2.97 \%$ among healthy volunteers $)(p=0.24)$. The frequency of MRSA among S. aureus was $27.77 \%$ (31.81\% in hospitalized patients and $21.42 \%$ among healthy volunteers). Methicillin-resistant Staphylococcus aureus was $100 \%$ and $66.67 \%$ resistant to norfloxacin and $57.14 \%$ and $33.33 \%$ resistant to erythromycin in patients and volunteers, respectively. However, gentamicin registered a sensitivity of $100 \%$. Gender, age, prior antibiotic use was not associated with carrying MRSA. Conclusion: This study on the prevalence of MRSA reports worrying results. There is an urgent need to develop measures to monitor the spread of these multidrug-resistant strains. Keywords: carriage, Healthy volunteers, Hospitalized patients, MRSA, Ouagadougou
\end{abstract}

\section{INTRODUCTION}

La résistance aux antibiotiques constitue une menace grave pour la santé mondiale et de fait impacte le développement. En effet elle entraîne une prolongation de l'hospitalisation des patients, une augmentation des dépenses de santé et une augmentation de la morbi-mortalité [1]. Les staphylocoques sont des bactéries retrouvées au niveau des narines antérieures en dehors de tout processus infectieux. On estime qu'environ un tiers de la population saine est porteur de Staphylococcus aureus dans les narines [2,3]. Staphylococcus aureus est impliqué souvent dans des infections communautaires et nosocomiales [4]. Il s'agit des infections cutanées bénignes comme les furoncles ou les panaris et des pathologies mettant en jeu le pronostic vital comme les septicémies, les endocardites, les pneumopathies et 
les infections du système nerveux central [5]. Par ailleurs, Staphylococcus. aureus est responsable de syndromes liés à l'action de ses toxines comme l'intoxication alimentaire et le syndrome du choc toxique [6]. Le portage asymptomatique de Staphylococcus aureus résistant à la méticilline(SARM) est associé à un risque élevé de développer une infection à SARM [7]. Il s'agit de ce fait d'un réel problème de santé publique car les traitements des infections associées à ces SARM font appel aux dernières molécules de l'arsenal thérapeutique contre les bactéries, souvent indisponibles dans les pays à ressources limitées. Les principaux facteurs de risque d'infection sont le portage nasal et toute rupture de la barrière cutanéomuqueuse favorisant la pénétration du germe. L'épidémiologie de Staphylococcus aureus a montré que l'émergence et la diffusion mondiale de SARM communautaires est liée à une expansion de clones spécifiques à chaque continent : Le ST300 est retrouvé majoritairement aux USA, le ST59 en Asie, et le ST80 est considéré comme le clone Européen. En Afrique de l'Ouest circulent principalement le ST5 et le ST15 [8]. La résistance à la méticilline montre des fréquences variant d'un pays à un autre mais tout en restant en général élevées allant de $16 \%$ à $39 \%$. Cela témoigne l'ampleur du problème des SARM dans la sousrégion. Au Burkina Faso une étude réalisée en 2016 à Bobo Dioulasso a rapporté un taux de $0 \%$ chez les volontaires sains, $3,9 \%$ chez les patients hospitalisés et une prévalence de $1,82 \%$ [8]. Cette présente étude de portage des SARM chez des volontaires sains ainsi que chez les malades hospitalisés a concerné la ville de Ouagadougou. Elle a porté sur la prévalence des SARM, les types de résistances et les principaux facteurs associés.

\section{Matériel et Méthodes}

Type, période et lieu de l'étude : Il s'agit d'une étude transversale prospective qui s'est déroulée du $1^{\text {er }}$ juillet au 30 novembre 2017 au centre hospitalier universitaire de Tengandogo à Ouagadougou.

Recrutements des patients et des volontaires : L'étude a concerné tous les patients hospitalisés pendant au moins $48 \mathrm{~h}$ et les volontaires sains (le personnel de santé et les accompagnants) rencontrés au centre hospitalier universitaire de Tengandogo pendant la période de l'étude.

Collecte des échantillons : Les prélèvements ont été faits par écouvillonnage des fosses nasales antérieures en tournant deux (2) fois l'écouvillon dans les deux narines [9]. Chaque écouvillon a été replacé dans son étui contenant $2 \mathrm{ml}$ d'eau physiologique stérile, puis acheminé immédiatement au laboratoire pour un enrichissement avant une analyse microbiologique.

Identification des bactéries : Chaque prélèvement nasal a été ensemencé immédiatement au bouillon nutritif (bouillon-cœur-cervelle) pendant 5 heures pour améliorer le rendement bactériologique [10].
Après cet enrichissement, $100 \mu \mathrm{l}$ du bouillon ont été repiqués sur la gélose Chapman. Les boîtes de Pétri ont été incubées à $37^{\circ} \mathrm{C}$ pendant 18 à 24 heures en aérobiose. La morphologie et le Gram, la présence de colonies sur Chapman, la fermentation du mannitol, la production de la catalase et de la coagulase ont servi à l'identification de Staphylococcus aureus.

Méthodes de détection des SARM : La détection de la résistance à la méticilline chez Staphylococcus aureus a été effectuée à l'aide du disque de céfoxitine (30 $\mu \mathrm{g})$ (Oxoid Ltd, Basingstone, $U K)$. Le diamètre de la zone d'inhibition a été mesuré à l'aide d'un pied à coulisse. Les diamètres inférieurs à $22 \mathrm{~mm}$ et/ou la présence de colonies dans la zone d'inhibition ont été retenus comme étant une résistance à la céfoxitine.

Détection des résistances associées : Elle a été réalisée selon la technique Kirby-Bauer en ensemençant par écouvillonnage une suspension en solution saline $(0,9 \% \mathrm{NaCl})$ équivalente au standard 0,5 Mc Farland. L'incubation a été effectuée à $37^{\circ} \mathrm{C}$ en aérobiose pendant 18 à 24 heures. La lecture et l'interprétation ont été effectuées selon les recommandations du comité d'antibiogramme de la Société Française de Microbiologie [11]. Les résistances associées des SARM, ont été faites en utilisant les disques norfloxacine $(10 \mu \mathrm{g})$, érythromycine $(15 \mu \mathrm{g})$, gentamicine $(30 \mu \mathrm{g})$ (HiMedia Ltd).

Contrôle qualité : Les procédures standards opérationnelles ont été strictement suivies. Les dates de péremption des milieux de cultures, des réactifs et autres consommables ont été vérifiées et documentées. Une vérification des contaminants des milieux déjà préparés a été effectuée. Un contrôle de qualité a été réalisé afin de tester la qualité des milieux. Chaque nouveau lot de disques a été contrôlé avant l'utilisation par une souche ATCC 25923 Staphylococcus aureus.

Considérations éthiques: Une autorisation du directeur général du centre hospitalier universitaire de Tengandogo a été obtenue avant le démarrage effectif de la collecte. Tous les sujets inclus dans notre étude ont été renseignés pour obtenir leur consentement éclairé par la version écrite. La participation a été libre. La confidentialité des informations obtenues sur les sujets de l'étude a été respectée, car les participants et leurs échantillons ont été codifiés.

Saisie et analyse des données : La saisie des données et les figures ont été réalisées sur le logiciel Excel 2013 et l'exploitation statistique des résultats à l'aide du logiciel XLSTAT 2017 version 19.5. Les répartitions des variables ont été comparées par le test $\chi^{2}$ d'indépendance. Le degré de signification des tests a été fixé à $5 \%$.

\section{Résultats}


Prévalence du portage nasal des SARM

Le taux de portage nasal de SARM était $7,95 \%$ et de $2,97 \%(p=0,24)$ respectivement chez les patients hospitalisés et chez les volontaires sains avec une prévalence globale de 5,29\%.

Resistances associées

La fréquence de SARM chez Staphylococcus aureus était $27,77 \%$ dont $31,81 \%$ chez les malades hospitalisés et $21,42 \%$ chez les volontaires sains. Les SARM étaient résistants à $100 \%$ à la pénicilline $\mathrm{G}$; à $100 \%$ et $66,67 \%$ à la norfloxacine et à $57,14 \%$ et $33,33 \%$ à l'érythromycine respectivement chez les patients hospitalisés et chez les volontaires. Cependant la sensibilité à la vancomycine et à la gentamicine était de $100 \%$ (Figure).

Analyse des facteurs associés

Les malades hospitalisés et les agents de santé étaient porteurs de SARM, mais les patients ont été les porteurs majoritaires des SARM (70\%) (Tableau I)

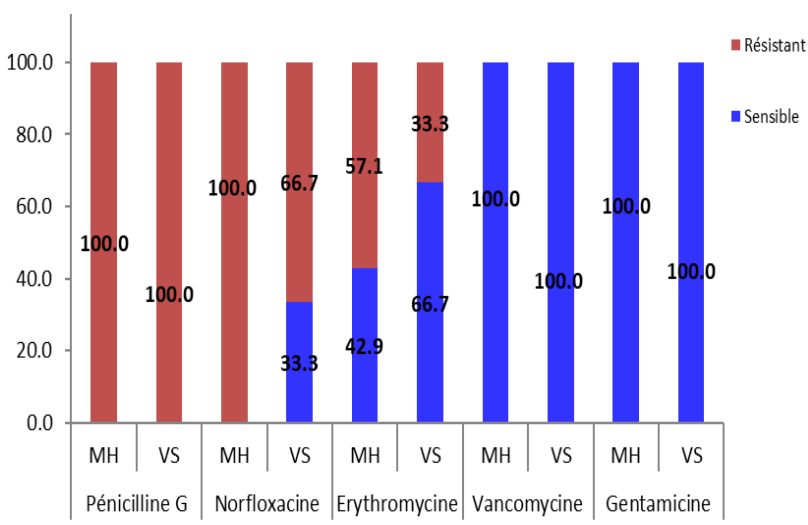

MH : malades hospitalisés, VS : volontaires sains

Figure 1 : Sensibilités des SARM aux antibiotiques

isolés parmi les patients et les volontaires

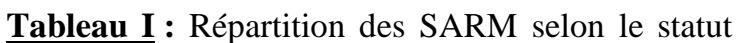
des inclus

\begin{tabular}{lcl}
\hline Statuts & $\begin{array}{c}\text { SARM } \\
+\end{array}$ & $\begin{array}{l}\text { Pource } \\
\text { ntage } \\
(\%)\end{array}$ \\
\hline Patients hospitalisés & 7 & 70,0 \\
Agents de santé & 3 & 30,0 \\
Accompagnants de malade & 0 & 0,0 \\
Total & $\mathbf{1 0}$ & $\mathbf{1 0 0 , 0}$ \\
\hline
\end{tabular}

Répartition des SARM selon les tranches d'âge Les patients âgés de 20 à 40 ans représentaient la moitié des porteurs de SARM (Tableau II)

Tableau II : Distribution des SARM selon les tranches d'âge

\begin{tabular}{|c|c|c|c|c|c|c|}
\hline Sujets & & $\begin{array}{l}\text { talisés } \\
\text { =88) }\end{array}$ & & $\begin{array}{l}\text { sains } \\
\text { 101) }\end{array}$ & \multirow[b]{3}{*}{$\mathbf{n}$} & \multirow[t]{3}{*}{ tal } \\
\hline \multirow{2}{*}{$\begin{array}{l}\text { Tranche } \\
\text { d'âge }\end{array}$} & \multicolumn{2}{|c|}{ SARM+ } & \multicolumn{2}{|c|}{ SARM+ } & & \\
\hline & $\mathbf{n}$ & $\%$ & $\mathbf{n}$ & $\%$ & & \\
\hline$\leq 20$ & 1 & 14,3 & 0 & 0,0 & 1 & 10,0 \\
\hline $21-40$ & 3 & 42,9 & 2 & 66,7 & 5 & 50,0 \\
\hline $41-59$ & 1 & 14,3 & 1 & 33,3 & 2 & 20,0 \\
\hline$\geq 60$ & 2 & 28,6 & 0 & 0,0 & 2 & 20,0 \\
\hline TOTAL & 7 & 100,0 & 3 & 100,0 & 10 & 100,0 \\
\hline
\end{tabular}

Répartition des SARM des patients selon le service

Les SARM ont été retrouvés en médecine (3), en chirurgie viscérale (2), en chirurgie traumatologie (1) et en néonatologie (1).

Analyses de l'association entre l'âge, le sexe, la notion de l'usage antérieur d'un antibiotique, les classes d'antibiotiques et le portage des SARM

La majorité des hommes étaient des patients hospitalisés (61,36\% vs 29,70\%, $p<0,0001)$. Cependant les femmes volontaires étaient majoritaires $(71,30 \%$ vs $38,64 \%, p<0,0001)$. L' analyse des facteurs d'association montre une différence significative entre l'usage antérieur des antibiotiques par des patients et des volontaires $(43,18 \%$ vs $26,73 \%, p=0,025)$. En fonction du type d'antibiotiques utilisés une différence significative a été obtenue pour les bêta-lactamines $(37,5 \%$ vs $18,81 \%, p=0,006)$ (Tableau III)

Tableau III : Caractéristiques de la population d'étude et analyses des facteurs associés

\begin{tabular}{lllll}
\hline Caractéristiques & $\begin{array}{c}\text { P. hospitalisés } \\
(\mathbf{n = 8 8})\end{array}$ & $\begin{array}{l}\text { V sains } \\
(\mathbf{n = 1 0 1})\end{array}$ & $\begin{array}{l}\text { Total } \\
(\mathbf{n = 1 8 9})\end{array}$ & $\boldsymbol{p}$-value \\
\hline moyenne d'âge (écart type) & $40,54(23,48)$ & $40,40(9,40)$ & $40,47(16,44)$ & \\
Homme n $(\%)$ & $54(61,36)$ & $30(29,70)$ & $84(44,44)$ & $<\mathbf{0 , 0 0 0 1}$ \\
Femme n $(\%)$ & $34(38,64)$ & $71(71,30)$ & $105(55,56)$ & $<\mathbf{0 , 0 0 0 1}$ \\
Usage antérieur d'ATB n(\%) & $38(43,18)$ & $27(26,73)$ & $65(34,39)$ & $\mathbf{0 , 0 2 5}$ \\
Portage de $\boldsymbol{S}$. aureus n $(\boldsymbol{\%})$ & $22(25)$ & $14(13,86)$ & $36(19,04)$ & 0,08 \\
Usage de $\boldsymbol{\beta}$-lactamines $\mathbf{n}(\boldsymbol{\%})$ & $33(37,50)$ & $19(18,81)$ & $52(27,51)$ & $\mathbf{0 . 0 0 6}$
\end{tabular}




\begin{tabular}{lllll} 
Usage de fluoroquinolones $\mathbf{n}(\boldsymbol{\%})$ & $1(1,14)$ & $7(6,93)$ & $8(4,23)$ & 0,08 \\
Autres antibiotiques $\mathbf{n}(\boldsymbol{\%})$ & $4(4,55)$ & $1(0,99)$ & $5(2,65)$ & 1,00 \\
\hline $\boldsymbol{P}:$ patients $; \boldsymbol{V}:$ volontaires & &
\end{tabular}

\section{Discussion \\ Prévalence des SARM}

Staphylococcus aureus est responsable de plusieurs infections cutanées et postopératoires. La prévalence globale de portage nasal de SARM était de 5,29\% dont 7,95\% chez les patients hospitalisés et $2,97 \%$ chez les volontaires. Cette prévalence du portage nasal pourrait augmenter la part des infections à SARM, mais aussi compliquer la prise en charge de ces infections. Nos résultats sont supérieurs à ceux rapportés par Ouédraogo et $a l$. en 2016 à Bobo Dioulasso, qui ont trouvé une prévalence globale de 1,82\% [12]. Cette différence résulterait de la variation des méthodes (technique d'échantillonnage, la technique de mise en culture, méthodes de détection des SARM). En effet nous avons effectué un enrichissement des écouvillons aux bouillons cœur-cervelle avant la mise en culture. Cet enrichissement semble augmenter la sensibilité de la détection en évitant les faux négatifs [10]. Cependant nos résultats se rapprochent du portage au niveau mondial des SARM qui varie entre $5,8 \%$ à $17,8 \%[10,13]$. Une étude réalisé en Angola en 2014 a montré une prévalence de 23,7\% [14].

Résistances associées des SARM aux antibiotiques isolés parmi les patients et les volontaires

La fréquence de portage de $S$. aureus était de $19,04 \%$ avec $27,77 \%$ de résistance à la méticilline. Chez les patients ces SARM étaient résistants à la pénicilline $\mathrm{G}(100 \%)$, à la norfloxacine $(100 \%)$ et à l'érythromycine $(57,1 \%)$. Par contre chez les volontaires sains ces SARM étaient résistants à la pénicilline $\mathrm{G}(100 \%)$, à la norfloxacine $(66,7 \%)$ et à l'érythromycine (33,3\%). Cependant la vancomycine et la gentamicine ont montré une efficacité de $100 \%$. Les SARM ont montré une résistance élevée aux fluoroquinolones et aux macrolides, de ce fait la vancomycine reste toujours l'antibiotique de choix pour les infections à SARM. En effet il a été prouvé aux USA que ces bactéries pourraient héberger plusieurs gènes de résistances qui leurs permettaient de résister non seulement aux bêta-lactamines, mais aussi aux fluoroquinolones, tétracyclines, macrolides, clindamycine et la mupirocine [15].

\section{Analyse des facteurs associés}

Les SARM ont été retrouvés dans toutes les tranches d'âge et dans les deux sexes. L'analyse des facteurs n'a pas pu être effectuée car les effectifs théoriques même après regroupement étaient inférieurs à cinq $(<5)$. Toutefois, les individus de la tranche d'âge de 20 à 40 étaient des porteurs majoritaires de SARM tant chez les patients que chez les volontaires, avec des fréquences respectives de $42,9 \%$ et $66,7 \%$. La limite de cette étude était qu'elle ne précisait pas le type de portage à savoir intermittent ou persistant. En effet, le portage persistant a un risque plus élevé d'autoinfection que le portage intermittent [16]. Nous avons obtenu une fréquence élevée de $S$. aureus chez les patients (25\%) ainsi que chez les volontaires sains $(13,86 \%)$. Cette fréquence élevée pourrait augmenter le risque de transmission interhumaine dans les hôpitaux du Burkina Faso.

\section{CONCLUSION}

Notre étude révèle que la prévalence de portage nasal des SARM est de 5,29\% avec 7,95\% chez les patients hospitalisés et 2,97\% parmi les volontaires sains. Aucun facteur associé au portage des SARM n’a été retrouvé. Des résistances associées existent aux fluoroquinolones et aux macrolides. Au vu de ces résultats et de la place qu'occupent les infections à bactéries multirésistantes dans les infections postopératoires et du tractus urinaire, il semble nécessaire d'instaurer une politique de surveillance des bactéries multi résistantes.

\section{Conflit d'intérêt : aucun}

\section{Contribution Des Auteurs}

Conception et design de l'étude : Soré S., Sanou I., Ouédraogo A.S., Ilboudo K.S., collecte et analyses des échantillons : Soré. S., Béogo S., Analyse des résultats: Soré S., Sanou I., Ouédraogo A.S., Sanou S., Dakouo S.N.P., Djamalladine M.D., Sawadogo Y., Tout les auteurs ont lu et approuvé le manuscrit final.

\section{Références}

1.Alabi OS, Sanusi EA. Efficacy of three disinfectant formulations against multidrug resistant nosocomial agents. African J Clin Exp Microbiol. 2012;13(3): 178-82.

2.Morgene MF. Modélisation in vitro de la colonisation nasale à Staphylococcus aureus ; interactions avec 1 ' infection à rhinovirus. Universitéde Lyon; 2018.

3.Kluytmans J, Belkum VA, Verbrugh H. Nasal Carriage of Staphylococcus aureus : Epidemiology, Underlying Mechanisms, and Associated Risks. Clin Microbiol Rev. 1997;10(3):505-20.

4.Yousef SAAL, Mahmoud SY, Taha M.

Prevalence of methicillin-resistant Staphylococcus aureus in Saudi Arabia: systemic review and metaanalysis. African J Clin Exp Microbiol.

2013;14(3):146-54.

5.Phillips CJ, Wells NA, Martinello M, Smith S, Woodman RJ, Gordon DL. Optimizing the detection of methicillin-resistant Staphylococcus aureus with elevated vancomycin minimum 
inhibitory concentrations within the susceptible range. Infect Drug Resist. 2016;9:87-92.

6.Leibler JH, León C, Cardoso LJP, Morris JC, Miller NS, Nguyen DD, et al. Prevalence and risk factors for MRSA nasal colonization among persons experiencing homelessness in Boston, MA. J Med Microbiol. 2017;66:1183-8.

7.Zouari A, Smaoui H, Hajji1 H, Chahed MK, Kechrid A. Prévalence du portage nasal de Staphylococcus aureus résistant à la méticilline chez 1 ' enfant Tunisien en milieu scolaire. Rev Tunisienne d'infectiologie. 2012;6(4):225-31. 8.Ouedraogo AS. Prévalence, circulation et caracterisation des bactéries multiresistantes au Burkina Faso. Collège Do. Université de Montpellier; 2016.

9.Riethmuller J. La résistance des entérobactéries aux carbapénèmes. Université de Strasbourg; 2013. 10.Lauderdale TY, Wang J, Lee W. Carriage rates of methicillin-resistant Staphylococcus aureus ( MRSA ) depend on anatomic location, the number of sites cultured, culture methods, and the distribution of clonotypes. Eur J Clin Microbiol Infect Dis. 2010;29:1553-9.

11.CA-SFM. Antibiogramm Committee of French Society for Microbiology. version 5. 2013. 1-63 p. 12.Ouedraogo A, Dunyach-remy C, Kissou A, Sanou S, Poda A, Kyelem C, et al. High Nasal
Carriage Rate of Staphylococcus aureus Containing Panton-Valentine leukocidin- and EDIN-Encoding Genes in Community and Hospital Settings in Burkina Faso. Front Microbiol.

2016;7(September):1-7.

13. Khanal R, Sah P, Lamichhane P, Lamsal A, Upadhaya S, Pahwa VK. Nasal carriage of methicillin resistant Staphylococcus aureus among health care workers at a tertiary care hospital in Western Nepal. Antimicrob Resist Infect Control. 2015;4(39):3-7.

14.Conceiçao T, Coelho C, Santos-silva I, Lencastre de H, Aires-de-Sousa M. Epidemiolgy of Methicillin-Resistant and Susceptible Staphylococcus aureus in Luanda, Angola: First Description of the Spread of the MRSA ST5-IVa. Microb Drug Resist. 2014;20(5):441-50. 15.Antri K, Rouzic N, Dauwalder O, Boubekri I, Bes M, Lina G, et al. High prevalence of methicillin-resistant Staphylococcus aureus clone ST80-IV in hospital and community settings in Algiers. Clin Microbiol Infect Dis. 2010;17:52632.

16.Muthukrishnan G, Lamers RP, Ellis A, Paramanandam V, Persaud AB, Tafur S, et al. Longitudinal genetic analyses of Staphylococcus aureus nasal carriage dynamics in a diverse population. BMC Infect Dis. 2013;13:1-13. 